\title{
Telemedicine for the management of COPD — near future or a hazy idea?
}

The authors declare no financial disclosure

Pneumonol Alergol Pol 2015; 83: 413-414

In this issue of PiAP we can read the invited systematic review on telemonitoring in COPD by Claudio Pedone and Diana Lelli from Università Campus Bio-Medico di Roma [1]. The authors have extraordinary experience in the field, they have published several papers on the topic, including a randomized controlled trial on the efficacy of telemonitoring in elderly patients with COPD [2]. In this study they showed reduced rate of exacerbations and COPD-related hospital admissions in patients followed up with the aid of a multiparametric remote monitoring system compared to patients followed up using the standard model of care. They concluded, that these results should be replicated and confirmed on a bigger cohort of COPD patients. This beneficial effect of telemonitoring was recently confirmed by Mierdel and Owen [3].

In the face of a shortage of big randomized trials, the statistical power to prove that telemedicine is efficacious and cost-effective could be improved by metaanalysis. So far at least two metaanalyses on this topic have been published [4, 5]. Polisena et al. [4] concluded, that home telehealth (home telemonitoring and telephone support) reduces rates of hospitalization and emergency department visits, while findings for hospital bed days of care varied between studies. Unexpectedly, the mortality rate was greater in the telephone-support group compared with usual care. Home telehealth interventions were similar or better than usual care for quality of life and patient satisfaction outcomes. Authors stress out the substantial heterogeneity of clinical outcomes measured by different authors, which made it difficult to draw unequivocal conclusions. Kitsiou et al. [5], who evaluated the potential role of telemonitoring in different chronic disorders (including COPD), point out that further efforts should be made to improve the design, conduct, reporting, and publication of systematic reviews and meta-analyses in this area. The current systematic review by Pedone and Lelli summarizes and updates the current knowledge in this particular field, however the conclusions are still the same. The authors say: "our study confirms that the available evidence on the effectiveness of telemedicine in COPD does not allow to draw definite conclusions; most evidence suggests a positive effect of telemonitoring on hospital admissions and ER visits. More trials with adequate sample size and with adequate consideration of background clinical services are needed to definitively establish its effectiveness".

What parameters should be monitored by telemedical systems in COPD patients? The list is almost endless. Starting from the most obvious clinical parameters like $\mathrm{FEV}_{1}$ or PEF measured by portable spirometer or PEF-meter, $\mathrm{SatO}_{2}$ measured by pulsoximeter, blood pressure, heart rate, through diurnal change of symptoms estimated by use of simple questionnaires, up to registration 
of behavioral changes, which may anticipate upcoming adverse event, i.e. disease exacerbation, need of hospital admission or even sudden death. Even an unexpected loss of compliance in data reporting may be a good measure of health status deterioration.

The next question is which patients should be subjected to telemonitoring first. Will it be potentially useful and economically justified to monitor all COPD patients? It seems reasonable to preselect with caution these patients who would benefit the most. Will it be a good solution for those, who exacerbate frequently? Or only those, who experience severe exacerbations or require hospitalization? Or only these patients with very high risk of death during upcoming exacerbation, i.e. with previous ICU admissions? In this case the destination group would be $\mathrm{C}$ and $\mathrm{D}$ category. But why not to monitor patients from category B, who have much more vascular comorbidities and the highest risk of death due to non-respiratory causes [6]. In this case, would it be reasonable in these patients to focus on cardiovascular rather, than respiratory parameters.

Different approaches have been proposed. Hernandez et al. [7] applied so-called Integrated Care Services in the project targeted to patients with COPD, cardiac failure and/or type 2 diabetes. This ambitious project included evaluation of: (1) enhanced care to prevent hospitalization, (2) home hospitalization and early discharge, (3) home-based maintenance of rehabilitation effects, and (4) support for remote diagnosis. The authors showed high potential of the four integrated care services to enhance health outcomes with cost-containment. This project indicated the possibility and even need of more complex and universal approach to telemonitoring of patients with multiple chronic coexistent disorders, however it also generated new methodological and practical problems.

Quite different approach was proposed by Blumenthal et al. [8], who compared in a randomized clinical trial coping skills training intervention (by helping them develop skills for coping more effectively with their disease) with COPD education for improving QoL and medical outcomes in COPD patients. The authors concluded, that a telehealth coping skills training intervention produced clinically meaningful improvements in quality of life and functional capacity, but no overall improvement in risk of COPD-related hospitalization and all-cause mortality. This study is an example, by what different means the goals could be achieved.
There are many unanswered or even unasked questions. Paradoxically, it is only a proof that we are at the very beginning of this journey. The standardization is the key issue.

Completely different application of telemedicine in pulmonology was proposed by Nowiński et al. (in this issue) [9]. The authors evaluated the use of spirometers with data transfer for training purposes. The telespirometric system was verified as a tool for spirometry training in family doctors' offices, and was found useful and effective.

It is obvious, that future will bring further development of IT technologies, and in consequence new and more technically and scientifically advanced techniques will be available for telemedicine. It is also of no doubt, that IT devices and services are becoming cheaper and cheaper. This is a good environment for the research on the application of these techniques in medicine. COPD, due to its prevalence and high costs generated for health care system is a natural candidate for this research. Let us move in the same pace with IT researchers.

\section{Conflict of interest}

The authors declare no conflict of interest.

\section{References:}

1. Pedone C, Lelli D. Systematic review of telemonitoring in COPD: an update. Pneumonol Alergol Pol 2015; 83: 476-8484. doi: 10.5603/PiAP.2015.0077

2. Pedone C, Chiurco D, Scarlata S, Incalzi RA. Efficacy of multiparametric telemonitoring on respiratory outcomes in elderly people with COPD: a randomized controlled trial. BMC Health Serv Res 2013; 13: 82. doi: 10.1186/1472-6963-13-82.

3. Mierdel S, Owen K. Telehomecare reduces ER use and hospitalizations at William Osler Health System. Global Telehealth 2015. doi: 10.3233/978-1-61499-505-0-102.

4. Polisena J, Tran K, Cimon K et al. Home telehealth for chronic obstructive pulmonary disease: a systematic review and meta-analysis. J Telemed Telecare 2010; 16: 120-127. doi: 10.1258/jtt.2009.090812.

5. Kitsiou S, Paré G, Jaana M. Systematic reviews and meta-analyses of home telemonitoring interventions for patients with chronic diseases: a critical assessment of their methodological quality. J Med Internet Res 2013; 15: e150. doi: 10.2196/jmir.2770.

6. de Torres JP, Casanova C, Marín JM et al. Prognostic evaluation of COPD patients: GOLD 2011 versus BODE and the COPD comorbidity index COTE. Thorax 2014; 69: 799-804. doi: 10.1136/thoraxjnl-2014-205770.

7. Hernandez C, Garcia-Aymerich J, Grimsmo A et al. Integrated care services: lessons learned from the deployment of the NEXEX project. Int J Integr Care 2015; URN:NBN:NL:UI:10-1-114811.

8. Blumenthal JA, Emery CF, Smith PJ et al. The effects of a telehealth coping skills intervention on outcomes in chronic obstructive pulmonary disease: primary results from the INSPIRE-II study. Psychosom Med 2014; 76: 581-592. doi: 10.1097/PSY.0000000000000101.

9. Nowiński A, Romański E, Bieleń P et al. Pilot program on distance training in spirometry testing - the technology feasibility study. Pneumonol Alergol Pol 2015; 83: 431-435. doi: 10.5603/PiAP.2015.0071 